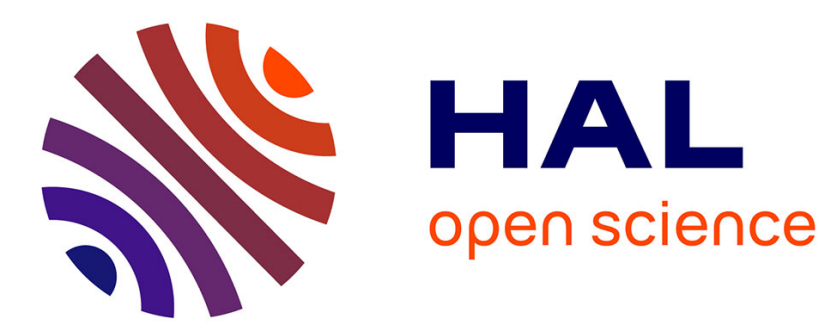

\title{
Davenport series and almost-sure convergence
}

Julien Brémont

\section{To cite this version:}

Julien Brémont. Davenport series and almost-sure convergence. Quarterly Journal of Mathematics, 2011, 62 (1), pp.825-843. hal-00794135

\section{HAL Id: hal-00794135 \\ https://hal.science/hal-00794135}

Submitted on 25 Feb 2013

HAL is a multi-disciplinary open access archive for the deposit and dissemination of scientific research documents, whether they are published or not. The documents may come from teaching and research institutions in France or abroad, or from public or private research centers.
L'archive ouverte pluridisciplinaire HAL, est destinée au dépôt et à la diffusion de documents scientifiques de niveau recherche, publiés ou non, émanant des établissements d'enseignement et de recherche français ou étrangers, des laboratoires publics ou privés. 


\title{
DAVENPORT SERIES AND ALMOST-SURE CONVERGENCE
}

\author{
Julien Brémont \\ Université Paris-Est, janvier 2010
}

\begin{abstract}
We consider Davenport-like series with coefficients in $l^{2}$ and discuss $L^{2}$-convergence as well as almost-everywhere convergence. We give an example where both fail to hold. We next improve former sufficient conditions under which these convergences are true.
\end{abstract}

\section{Introduction}

Let $\mathbb{R} \backslash \mathbb{Z}$ be the Circle and $L^{2}$ be the restriction of the space $L^{2}(\mathbb{R} \backslash \mathbb{Z} \rightarrow \mathbb{R})$ to odd functions. For a real parameter $\lambda>1 / 2$, we introduce the map :

$$
g_{\lambda}(x)=\sum_{m \geq 1} \frac{\sin (2 \pi m x)}{m^{\lambda}} .
$$

This function is defined everywhere on $\mathbb{R} \backslash \mathbb{Z}$. It is continuous, except at 0 when $1 / 2<\lambda \leq 1$, and belongs to $L^{2}$. For real sequences $\left(a_{n}\right) \in l^{2}$, we consider expansions based on the dilated functions system $\left\{g_{\lambda}(n x)\right\}_{n \geq 1}$ of the following form :

$$
\sum a_{n} g_{\lambda}(n x)
$$

where we write $\sum$ for the summation $\sum_{n=1}^{+\infty}$. We are interested in the questions of $L^{2}$-convergence and Lebesgue almost-everywhere (a.e) convergence of such series.

This is a natural problem which can be formulated with $g_{\lambda}$ replaced by a general $g \in L^{2}(\mathbb{R} \backslash \mathbb{Z})$. A reason for focusing on odd functions is that sin series in general better converge than cos series. When $g(x)=\sin (2 \pi x)$, the $L^{2}$-convergence of $\sum a_{n} g(n x)$ follows from the fact that the $\{g(n x)\}_{n \geq 1}$ are orthonormal. A.e-convergence in this case is the difficult theorem of Carleson [4]. For a different $g$ such that the $\{g(n x)\}_{n \geq 1}$ are complete in $L^{2}$, the $\{g(n x)\}_{n \geq 1}$ are not orthogonal, see Bourgin and Mendel [2], and the question of $L^{2}$-convergence is already not clear. The case of $g=g_{\lambda}$ was introduced by Wintner in [17]. A special motivation comes Arithmetics and the case $\lambda=1$, corresponding to the first Bernoulli polynomial or "sawtooth function" $\{x\}:=x-[x]-1 / 2$, where $[x]$ is the integer part of $x \in \mathbb{R}$. Indeed :

$$
\{x\}=-\frac{1}{\pi} \sum_{m \geq 1} \frac{\sin (2 \pi m x)}{m} .
$$

Series of the form $\sum a_{n}\{n x\}$ appear since long ago in the litterature, at the interface of Number Theory and Analysis. We recommend the very detailed presentation of such series by Jaffard in [12], where they are called Davenport series, due to Davenport's initial systematic study $[5,6]$. In

AMS 2000 subject classifications : 11A25, 26A30, 42A16, 42A61, 60F05

Key words and phrases : Davenport series, Carlitz's lemma, $L^{2}$-convergence, almost-everywhere convergence. 
this article and for simplicity we call $D_{\lambda}$-series a series of the form (1), the case of Davenport series corresponding to $\lambda=1$.

Beginning with a discussion in $L^{2}$, Wintner [17] established that the family $\left\{g_{\lambda}(n x)\right\}_{n \geq 1}$ is complete in $L^{2}$ for any $\lambda>1 / 2$. We now consider the $L^{2}$-convergence of $D_{\lambda}$-series with $\left(a_{n}\right) \in l^{2}$. According to work by Wintner [17] and next Hedenmalm, Lindqvist and Seip [11], the $\left\{g_{\lambda}(n x)\right\}_{n \geq 1}$ form a Riesz basis when $\lambda>1$. By a "Riesz basis" we mean a complete sequence $\left(\xi_{n}\right)$ in $L^{2}$ such that for some constant $C>0$ :

$$
C^{-1} \sum a_{n}^{2} \leq\left\|\sum a_{n} \xi_{n}\right\|^{2} \leq C \sum a_{n}^{2}, \forall\left(a_{n}\right) \in l^{2} .
$$

Here and in the whole article we denote by \|\| the usual $L^{2}$-norm, with scalar product $\langle$,$\rangle . Lindqvist$ and Seip [15] provide the inequalities :

$$
\frac{\zeta(2 \lambda)}{\zeta(\lambda)^{2}} \sum a_{n}^{2} \leq\left\|\sum a_{n} g_{\lambda}(n x)\right\|^{2} \leq \frac{\zeta(\lambda)^{2}}{\zeta(2 \lambda)} \sum a_{n}^{2},
$$

where $\zeta(s)=\sum_{n \geq 1} n^{-s}$, for $s>1$, is the Riemann Zeta function. Constants are optimal. This settles the question of $L^{2}$-convergence when $\lambda>1$. In this case, the a.e-convergence of $D_{\lambda}$-series for $\left(a_{n}\right) \in l^{2}$ follows from Carleson's theorem [4] on the a.e-convergence of Fourier series. Indeed :

$$
\sum_{n=1}^{N} a_{n} g_{\lambda}(n x)=\sum_{m \geq 1} m^{-\lambda} \sum_{n=1}^{N} a_{n} \sin (2 \pi m n x) .
$$

For each $m \geq 1, \sum_{n=1}^{N} a_{n} \sin (2 \pi m n x)$ converges a.e, as $N \rightarrow+\infty$, by Carleson's theorem. Next :

$$
\left|\sum_{n=1}^{N} a_{n} \sin (2 \pi m n x)\right| \leq \sup _{K \geq 1}\left|\sum_{n=1}^{K} a_{n} \sin (2 \pi m n x)\right|=: M(m x) .
$$

By classical work on the maximal operator, $\|M\|_{L^{1}(\mathbb{R} \backslash \mathbb{Z})} \leq C \sum a_{n}^{2}$ (cf for instance Fefferman [7]). Thus $\sum_{m>1} m^{-\lambda} M(m x)$ is integrable and in particular a.e finite. One can now a.e apply the Lebesgue dominated convergence theorem in (2) to conclude. Of course this argument does not work when $1 / 2<\lambda \leq 1$. Mention also that Carleson's theorem uses properties of the Fourier basis. There exists orthonormal bases of $L^{2}$ for which $L^{2}$-convergence does not imply a.e-convergence, see Rademacher [16].

For the rest of the article we suppose that $1 / 2<\lambda \leq 1$. As a consequence of an analysis by Wintner [17] of some Dirichlet series associated to $D_{\lambda}$-series, for any $1 / 2<\lambda \leq 1$ there exists $\left(a_{n}\right) \in l^{2}$ such that $\sum a_{n} g_{\lambda}(n x)$ is $L^{2}$-divergent. In particular for $1 / 2<\lambda \leq 1$, the $\left\{g_{\lambda}(n x)\right\}_{n \geq 1}$ do not form a Riesz basis of $L^{2}$. We now detail known sufficient conditions for $L^{2}$ and a.e-convergence. We are essentially aware of results concerning Davenport series. Wintner [17] proved that $\sum a_{n}\{n x\}$ converges in $L^{2}$ whenever $a_{n}=O\left(n^{-\kappa}\right)$, with $\kappa>1 / 2$. Extending this result, Jaffard [12] showed that for $\left(a_{n}\right) \in l^{2}$ the sum $\sum a_{n}\{n x\}$ converges in a Sobolev space very close to $L^{2}$. About a.econvergence, Davenport in his fundamental papers $[5,6]$ gave non-trivial arithmetical examples where a.e-convergence is true, such as :

$$
\sum_{n=1}^{+\infty} \frac{\lambda(n)}{n}\{n x\}, \sum_{n=1}^{+\infty} \frac{\Lambda(n)}{n}\{n x\}, \sum_{n=1}^{+\infty} \frac{\mu(n)}{n^{2}}\left\{n^{2} x\right\},
$$

where $\lambda(n), \Lambda(n)$ and $\mu(n)$ are respectively Liouville's function, Von Mangolt's function and Mobius' function. When the $a_{n}$ are slowly varying, the a.e-convergence of $\sum a_{n}\{n x\}$ follows, via an Abel transform, from estimates on $\sum_{n<N}\{n x\}$, cf Lang [14]. Jaffard [12] deduced the a.econvergence of $\sum a_{n}\{n x\}$, whenever $a_{n}=O(\log n)^{-\alpha}$ and $a_{n+1}-a_{n}=O\left(n^{-1}(\log n)^{-(1+\alpha)}\right)$ for some $\alpha>2$. In particular, Hecke series : 


$$
\mathcal{H}_{s}(x)=\sum_{n=1}^{+\infty} \frac{\{n x\}}{n^{s}}
$$

converge a.e for $\operatorname{Re}(s)>0$, a result already shown by Hardy and Littlewood [8]. For general sequences, Hartman proved in [9] the a.e-convergence of $\sum a_{n}\{n x\}$ when $a_{n}=O\left(n^{-\kappa}\right)$, with $\kappa>2 / 3$. Mention finally some results going further than a.e-convergence. Using P-summation techniques, de la Bretêche and Tenenbaum [3] proved that (4) is convergent when $s=1$ outside a set of Hausdorff dimension zero that they describe. Also the second series in (3) is everywhere convergent.

We now detail the content of the article. We discuss the $L^{2}$ and a.e-convergence of $D_{\lambda}$-series of the form (1) for general $\left(a_{n}\right) \in l^{2}$. We first complete the $L^{2}$-divergence result of Wintner [17] by an a.e-divergence result. We next improve former sufficient conditions for $L^{2}$ and a.e-convergence.

\section{Theorem 1.1}

Assume that $1 / 2<\lambda \leq 1$.

i) There exists $\left(a_{n}\right) \in l^{2}$ such that $\sum a_{n} g_{\lambda}(n x)$ is simultaneously $L^{2}$-divergent and a.e-divergent.

ii) Suppose that for some $\varepsilon>0$ :

$$
\left\{\begin{array}{c}
\sum a_{n}^{2} n^{\frac{(1+\varepsilon)(\log n)^{-}(2 \lambda-1)}{2(1-\lambda) \log \log n}}<\infty, \text { when } 1 / 2<\lambda<1, \\
\sum a_{n}^{2}(\log n)^{3}(\log \log n)^{2+\varepsilon}<\infty, \text { when } \lambda=1 .
\end{array}\right.
$$

Then $\sum a_{n} g_{\lambda}(n x)$ converges in $L^{2}$ and a.e.

In particular, the latter conditions are verified if $\sum a_{n}^{2} n^{\varepsilon}<\infty$, for some $\varepsilon>0$. For example, the following series converge in $L^{2}$ and a.e when $s>1 / 2$ :

$$
\sum_{n=1}^{+\infty} \frac{\lambda(n)}{n^{s}}\{n x\}, \sum_{n=1}^{+\infty} \frac{\Lambda(n)}{n^{s}}\{n x\} \text { and } \sum_{n=1}^{+\infty} \frac{\mu(n)}{n^{2 s}}\left\{n^{2} x\right\} .
$$

We note that whereas Wintner's approach is abstract we build here an explicit example. The fact that $\left(a_{n}\right) \in l^{2}$ does not imply the a.e-convergence of $D_{\lambda}$-series is not that surprising, since this condition is not the correct one for $L^{2}$-convergence. One can make the second moment explode and develop a probabilistic argument based on the Central Limit Theorem. The true question, more difficult, is whether $L^{2}$-convergence implies a.e-convergence. A weak formulation is as follows :

Question. If $1 / 2<\lambda \leq 1$ and $\sum_{k \geq 1}\left(\sum_{n \geq 1} n^{-\lambda}\left|a_{k n}\right|\right)^{2}<+\infty$, does $\sum a_{n} g_{\lambda}(n x)$ converge a.e?

The above condition is strictly stronger than $\left(a_{n}\right) \in l^{2}$, when $1 / 2<\lambda \leq 1$. As detailed below, it ensures the $L^{2}$-convergence of $\sum a_{n} g_{\lambda}(n x)$ and is necessary when the $a_{n}$ have constant sign.

We next consider three classical situations, for instance Hadamard lacunarity, where we can prove $L^{2}$-convergence, but a.e-convergence only under stronger conditions. We define the support $\operatorname{supp}(n)$ of an integer $n$ as its set of prime divisors and write $|\operatorname{supp}(n)|$ for the cardinal of this set.

\section{Theorem 1.2}

Suppose that $1 / 2<\lambda \leq 1$.

i) Let $\left(n_{k}\right)$ check $n_{k+1} / n_{k} \geq c$, where $c>1$. Then $\sum a_{k} g_{\lambda}\left(n_{k} x\right)$ converges in $L^{2}$ whenever $\left(a_{k}\right) \in l^{2}$ and more precisely:

$$
C_{1} \sum a_{k}^{2} \leq\left\|\sum a_{k} g_{\lambda}\left(n_{k} x\right)\right\|^{2} \leq C_{2} \sum a_{k}^{2}
$$


where :

$$
C_{1}=(1-1 / e) \frac{\zeta(4 \lambda)}{2}\left(\frac{2 \lambda-1}{2 \lambda}\right)\left(\frac{\ln \left(c^{2 \lambda-1}\right)}{1+\ln \left(c^{2 \lambda-1}\right)}\right)^{2} \text { and } C_{2}=\frac{\zeta(2 \lambda)}{2}\left(\frac{c^{\lambda}+1}{c^{\lambda}-1}\right) .
$$

If the stronger condition $\sum a_{k}^{2}(\log k)^{2}<\infty$ is verified, then $\sum a_{k} g_{\lambda}\left(n_{k} x\right)$ is also a.e-convergent.

ii) If $\left(a_{n}\right) \in l^{2}$ and $\left\{|\operatorname{supp}(n)|, a_{n} \neq 0\right\}$ is finite, then $\sum a_{n} g_{\lambda}(n x)$ converges in $L^{2}$. In fact, for $N \geq 1$ there exists $C(\lambda, N)>0$ such that for $\left(a_{n}\right) \in l^{2}$ with $a_{n}=0$ if $|\operatorname{supp}(n)|>N$, then:

$$
C^{-1}(\lambda, N) \quad \sum a_{n}^{2} \leq\left\|\sum a_{n} g_{\lambda}(n x)\right\|^{2} \leq C(\lambda, N) \sum a_{n}^{2} .
$$

If moreover $\sum a_{n}^{2}(\log n)^{2}<\infty$, then $\sum a_{n} g_{\lambda}(n x)$ is also a.e-convergent.

iii) Let $a_{n}=O\left(b_{n}\right)$, where $\left(b_{n}\right)_{n \geq 1} \in l^{2} \cap\left(\mathbb{R}_{+}\right)^{\mathbb{N}}$ satisfies $b_{n m}=b_{n} b_{m}$ whenever $n$ and $m$ are relatively prime. Then $\sum a_{n} g_{\lambda}(n x)$ converges in $L^{2}$.

A word on the method. The study of the convergence of Davenport series often starts with trying to write $\sum a_{n}\{n x\}$ as a Fourier series $\sum c_{m} \sin 2 \pi m x$. It was indeed remarked by Davenport [5] that formally the $\left(c_{m}\right)$ are explicitly given in terms of the $\left(a_{n}\right)$ and vice-versa. An alternative approach, developed here, when considering $L^{2}$-convergence is to orthonormalize the $\left\{g_{\lambda}(n x)\right\}_{n \geq 1}$. The Gram-Schmidt orthonormalisation procedure is explicit and a consequence of Carlitz's lemma on the reduction of quadratic forms. We provide details for simplicity. This furnishes a rather simple characterization of $L^{2}$-convergence. Theorem 1.2 follows via more or less standard computations. Concerning a.e-convergence, the orthonormalisation approach allows to adapt a technique of Rademacher [16] initially developed for the pointwise convergence of series built with general orthonormal systems.

A few notations. We write $i \wedge j$ and $i \vee j$ respectively for the greatest common divisor and the smallest common multiple of integers $i$ and $j$. The set of primes is $\mathcal{P}=\left\{p_{n}, n \geq 1\right\}$.

\section{Orthonormalization}

Recall that $1 / 2<\lambda \leq 1$. We first study correlations. The following computation is already contained in Lindqvist and Seip [15].

\section{Lemma 2.1}

Let $i, j \geq 1$. Then $\left\langle g_{\lambda}(i),. g_{\lambda}(j).\right\rangle=\frac{\zeta(2 \lambda)}{2}\left(\frac{i \wedge j}{i \vee j}\right)^{\lambda}$.

Proof of the lemma:

Let $i^{\prime}=i / i \wedge j, j^{\prime}=j / i \wedge j$. Since Lebesgue measure on $\mathbb{R} \backslash \mathbb{Z}$ is invariant by $x \longmapsto p x$ for any integer $p$, we have $\left\langle g_{\lambda}(i),. g_{\lambda}(j).\right\rangle=\left\langle g_{\lambda}\left(i^{\prime}.\right), g_{\lambda}\left(j^{\prime}.\right)\right\rangle$. Using the Fourier expansion of $g_{\lambda}$ :

$$
\left\langle g_{\lambda}\left(i^{\prime} .\right), g_{\lambda}\left(j^{\prime} .\right)\right\rangle=\sum_{k, l \geq 1} \int_{0}^{1} \frac{\sin \left(2 \pi k i^{\prime} x\right)}{k^{\lambda}} \frac{\sin \left(2 \pi l j^{\prime} x\right)}{l^{\lambda}} d x=\frac{1}{2} \sum_{m \geq 1} \frac{1}{\left(m^{2} i^{\prime} j^{\prime}\right)^{\lambda}}=\frac{\zeta(2 \lambda)}{2}\left(i^{\prime} j^{\prime}\right)^{-\lambda},
$$

since a relation $k i^{\prime}=l j^{\prime}$ reduces to $k=j^{\prime} m$ and $l=i^{\prime} m$ for some integer $m$. This concludes the proof of the lemma.

Remark. - The correlations being positive, if $\sum b_{n} g_{\lambda}(n x)$ is $L^{2}$-convergent with $\left(b_{n}\right) \in\left(\mathbb{R}_{+}\right)^{\mathbb{N}}$ and if $a_{n}=O\left(b_{n}\right)$, then $\sum a_{n} g_{\lambda}(n x)$ also converges in $L^{2}$.

We turn to the orthonormalization of the $\left\{g_{\lambda}(n x)\right\}_{n \geq 1}$. The following proposition is an application of Carlitz's lemma, cf for instance Haukkanen, Wang and Sillanp [10]. 
Recall that the Möbius function $\mu$ on the integers is defined by $\mu(1)=1, \mu\left(p_{i_{1}} \cdots p_{i_{k}}\right)=(-1)^{k}$ and $\mu(n)=0$ if $n$ is not square-free. If $f$ and $g$ are real maps defined on the integers related by $f(n)=\sum_{k \mid n} g(k)$, then (Möbius inversion formula) we have $g(n)=\sum_{k \mid n} \mu(n / k) f(k)$.

\section{Proposition 2.2}

i) Let $f_{n, \lambda}(x)=n^{-\lambda} \sum_{k \mid n} k^{\lambda} \mu(n / k) g_{\lambda}(k x), n \geq 1$. Then $\left\{f_{n, \lambda}\right\}_{n \geq 1}$ is an orthogonal family with :

$$
\left\|f_{n, \lambda}\right\|^{2}=\frac{\zeta(2 \lambda)}{2} \prod_{p \mid n, p \in \mathcal{P}}\left(1-p^{-2 \lambda}\right) \in\left(\frac{1}{2}, \frac{\zeta(2 \lambda)}{2}\right) .
$$

The $\left\{f_{n, \lambda}\right\}_{n \geq 1}$ form an orthogonal Riesz basis of $L^{2}$, with :

$$
2 \zeta(2 \lambda)^{-1} \sum_{n \geq 1}\left\langle f_{n, \lambda}, h\right\rangle^{2} \leq\|h\|^{2} \leq 2 \sum_{n \geq 1}\left\langle f_{n, \lambda}, h\right\rangle^{2}, \forall h \in L^{2} .
$$

ii) An equality $\sum_{i=1}^{n} a_{i} g_{\lambda}(i)=.\sum_{i=1}^{n} b_{i} f_{i, \lambda}$ holds if and only if $b_{i}=\sum_{k=1}^{[n / i]} k^{-\lambda} a_{k i}, 1 \leq i \leq n$. These equalities are reversed into $a_{i}=\sum_{k=1}^{[n / i]} k^{-\lambda} \mu(k) b_{k i}, 1 \leq i \leq n$.

Proof of the proposition:

Let $n \geq 1$. We introduce $n$-square matrices $D$ and $T$, where $D$ is diagonal and $T$ is upper-triangular. Set $T=\left(t_{i j}\right)$ with $t_{i j}=j^{-\lambda} 1_{i \mid j}$ and write $D=\operatorname{diag}\left(d_{i}\right)$, where the $\left(d_{i}\right)$ are defined below. First :

$$
\left({ }^{t} T D T\right)_{i j}=\sum_{1 \leq k \leq n} t_{k i} d_{k} t_{k j}=(i j)^{-\lambda} \sum_{k \mid i \wedge j} d_{k} .
$$

We choose $D$ so that $\sum_{k \mid m} d_{k}=(\zeta(2 \lambda) / 2) m^{2 \lambda}$, which by the Möbius inversion formula corresponds to setting $d_{k}=(\zeta(2 \lambda) / 2) \sum_{l \mid k} \mu(k / l) l^{2 \lambda}$. Lemma 2.1 gives $\left({ }^{t} T D T\right)_{i j}=\left\langle g_{\lambda}(i),. g_{\lambda}(j).\right\rangle$.

Next, the inverse of $T$ is given by $T_{i j}^{-1}=1_{i \mid j} i^{\lambda} \mu(j / i)$, since for any $i \leq j$ :

$$
\sum_{k=1}^{n} 1_{i \mid k} i^{\lambda} \mu(k / i) j^{-\lambda} 1_{k \mid j}=1_{i \mid j} i^{\lambda} j^{-\lambda} \sum_{l \mid j / i} \mu(l)=1_{i=j}
$$

using that $\sum_{k \mid m} \mu(k)=0$, if $m \geq 2$. Observe that $f_{i, \lambda}=i^{-\lambda} \sum_{1 \leq k \leq n}\left({ }^{t} T^{-1}\right)_{i k} g_{\lambda}(k$. $)$, for $1 \leq i \leq n$. The $\left\{f_{i, \lambda}\right\}_{i \geq 1}$ are therefore orthogonal in $L^{2}$, with $\left\|f_{i, \lambda}\right\|^{2}=d_{i} i^{-2} \lambda$. They also form a complete family, since it is the case for the $\left\{g_{\lambda}(n x)\right\}$, cf Wintner [17]. Decomposing $i=p_{l_{1}}^{\alpha_{1}} \cdots p_{l_{k}}^{\alpha_{k}}$ in prime factors, we have :

$$
\left\|f_{i, \lambda}\right\|^{2}=\frac{\zeta(2 \lambda)}{2} \sum_{d \mid i} d^{-2 \lambda} \mu(d)=\frac{\zeta(2 \lambda)}{2} \prod_{j=1}^{k} \sum_{d \mid p_{l_{j}}} d^{-2 \lambda} \mu(d)=\frac{\zeta(2 \lambda)}{2} \prod_{p \mid i, p \in \mathcal{P}}\left(1-p^{-2 \lambda}\right)
$$

Finally :

$$
\sum_{i=1}^{n} a_{i} g_{\lambda}(i .)=\sum_{i=1}^{n} a_{i} \sum_{k=1}^{n}\left({ }^{t} T\right)_{i k} k^{\lambda} f_{k, \lambda}=\sum_{i=1}^{n} a_{i} \sum_{k=1}^{n} i^{-\lambda} 1_{k \mid i} k^{\lambda} f_{k, \lambda}=\sum_{k=1}^{n} f_{k, \lambda} \sum_{i=1}^{[n / k]} i^{-\lambda} a_{k i} .
$$

The reversed formula is proved in a similar way.

We deduce the following characterization of $L^{2}$-convergence. 


\section{Corollary 2.3}

i) The series $\sum a_{n} g_{\lambda}(n x)$ converges in $L^{2}$ if and only if the numerical series $\sum_{k \geq 1} k^{-\lambda} a_{k i}$ converge for all $i \geq 1$, together with the uniformity condition :

$$
\sum_{i \geq 1}\left(\sum_{k>[n / i]} k^{-\lambda} a_{k i}\right)^{2} \rightarrow 0, \text { as } n \rightarrow \infty .
$$

ii) A sufficient condition for $\sum a_{n} g_{\lambda}(n x)$ to be $L^{2}$-convergent is :

$$
\sum_{i \geq 1}\left(\sum_{k \geq 1} k^{-\lambda}\left|a_{k i}\right|\right)^{2}<+\infty .
$$

This condition is necessary when $\left(a_{n}\right) \in\left(\mathbb{R}_{+}\right)^{\mathbb{N}}$.

Proof of the corollary:

If $\sum a_{n} g_{\lambda}(n x)$ converges in $L^{2}$, by proposition 2.2 the component $\sum_{k=1}^{[n / i]} k^{-\lambda} a_{k i}$ with respect to each $f_{i, \lambda}$ converges as $n \rightarrow+\infty$. The $L^{2}$-limit then has to be $\sum_{i>1} f_{i, \lambda}\left(\sum_{k>1} k^{-\lambda} a_{k i}\right)$. The uniformity condition is a consequence from the fact that the norm of $f_{i, \lambda}$ belongs to $(1 / 2, \zeta(2 \lambda) / 2)$. The first assertion of the second item is an application of the Lebesgue Dominated Convergence Theorem, whereas the second one follows from the first item.

Remark. - Corollary 2.3 can also be obtained when considering directly the Fourier expansion of $\sum a_{n} g_{\lambda}(n x)$ given by $g_{\lambda}$. In the sequel, the orthonormalization point of view has the practical advantage to keep finite all partial sums.

\section{A $l^{2}$-example of a $L^{2}$-divergent and a.e-divergent series}

We prove theorem $1.1 i$ ), using that for $1 / 2<\lambda \leq 1$ the series $\sum_{p \in \mathcal{P}} p^{-\lambda}$ is divergent. For each integer $K \geq 1$, we choose a finite set $\mathcal{P}_{K}=\left\{p_{j, K}\right\}_{1 \leq j \leq l_{K}}$ of consecutive primes satisfying :

$$
\sum_{j=1}^{l_{K}}\left(p_{j, K}\right)^{-\lambda} \geq K
$$

We fix $m_{K} \geq 2$ so that $\left(\frac{m_{K}-1}{m_{K}}\right)^{l_{K}} \geq 1 / 2$. Introduce sets :

$$
\left\{\begin{array}{c}
F_{1, K}=\left\{p_{1, K}^{u_{1}} \cdots p_{l_{K}, K}^{u_{l_{K}}}, 1 \leq u_{1}, \cdots, u_{l_{K}} \leq m_{K}\right\} \\
F_{1, K}^{\prime}=\left\{p_{1, K}^{u_{1}} \cdots p_{l_{K}, K}^{u_{l_{K}}}, 1 \leq u_{1}, \cdots, u_{l_{K}} \leq m_{K}-1\right\} .
\end{array}\right.
$$

We have $\left|F_{1, K}\right|=\left(m_{K}\right)^{l_{K}}$ and $\left|F_{1, K}^{\prime}\right|=\left(m_{K}-1\right)^{l_{K}}$. Let $q_{1, K}=1$ and take next infinitely many primes $q_{2, K}<\cdots<q_{n, K}<\cdots$, larger than $p_{l_{K}, K}$ and subject to the condition :

$$
\left(p_{1, K} \cdots p_{l_{K}, K}\right)^{m_{K}}\left(1+\frac{\left(m_{K}\right)^{l_{K} / 2}}{K}\right) \sum_{r=2}^{+\infty} \frac{\left(q_{r-1, K}\right)^{r-1}}{q_{r, K}} \leq \frac{1}{K} .
$$

Define the random variable :

$$
X_{1, K}=\frac{1}{K\left|F_{1, K}\right|^{1 / 2}} \sum_{n \in F_{1, K}} g_{\lambda}(n x) .
$$


It has zero mean and belongs to $L^{2}$. We write $\sigma_{K}^{2}=\int_{0}^{1}\left(X_{1, K}\right)^{2}(x) d x$ for its variance and choose an integer $T_{K} \geq K$ so that:

$$
\int_{0}^{1}\left(X_{1, K}\right)^{2}(x) 1_{\left\{\left|X_{1, K}\right|>\left(T_{K}\right)^{1 / 12} \sigma_{K}\right\}} d x \leq \frac{1}{K} .
$$

We define another collection of sets :

$$
\left\{\begin{array} { l } 
{ F _ { 2 , K } = q _ { 2 , K } F _ { 1 , K } } \\
{ F _ { 2 , K } ^ { \prime } = q _ { 2 , K } F _ { 1 , K } ^ { \prime } }
\end{array} \cdots \left\{\begin{array}{l}
F_{T_{K}, K}=q_{T_{K}, K} F_{1, K} \\
F_{T_{K}, K}^{\prime}=q_{T_{K}, K} F_{1, K}^{\prime}
\end{array}\right.\right.
$$

Grouping sets, we define :

$$
E_{K}=\bigcup_{r=1}^{T_{K}} F_{r, K} \text { and } E_{K}^{\prime}=\bigcup_{r=1}^{T_{K}} F_{r, K}^{\prime}
$$

We have $\left|E_{K}\right|=T_{K}\left|F_{1, K}\right|$ and $\left|E_{K}^{\prime}\right|=T_{K}\left|F_{1, K}^{\prime}\right|$. In particular :

$$
\frac{\left|E_{K}^{\prime}\right|}{\left|E_{K}\right|}=\frac{\left|F_{1, K}^{\prime}\right|}{\left|F_{1, K}\right|}=\left(\frac{m_{K}-1}{m_{K}}\right)^{l_{K}} \geq \frac{1}{2} .
$$

When considering the next integer (ie $K+1$ ) we start with $p_{1, K+1}>q_{T_{K}, K}\left(p_{1, K} \cdots p_{l_{K}, K}\right)^{m_{K}}$. Observe that all the $\left(F_{r, K}\right)_{K \geq 1,1 \leq r \leq T_{K}}$, are pairwise disjoint and in particular the $\left(E_{K}\right)_{K \geq 1}$, which furthermore are consecutive. We finally set :

$$
a_{n}=\left\{\begin{array}{cc}
\frac{1}{K\left|E_{K}\right|^{1 / 2}} & , \text { when } n \in E_{K}, \text { for some } K \geq 1, \\
0 & \text {, otherwise. }
\end{array}\right.
$$

This completes the definition of the sequence $\left(a_{n}\right)$. Formally $\sum a_{n} g_{\lambda}(n x)=\sum_{K \geq 1} Z_{K}$, with :

$$
Z_{K}=\frac{1}{\sqrt{T_{K}}} \sum_{r=1}^{T_{K}} X_{r, K}(x) \text { and } X_{r, K}(x)=\frac{1}{K\left|F_{1, K}\right|^{1 / 2}} \sum_{n \in F_{r, K}} g_{\lambda}(n x) \text {. }
$$

From the previous construction, observe that a partial sum $\sum_{K=1}^{N} Z_{K}(x)$ corresponds to a partial sum of $\sum a_{n} g_{\lambda}(n x)$. We now proceed to verifications.

i) The sequence $\left(a_{n}\right)$ belongs to $l^{2}$. Indeed :

$$
\sum_{n \geq 1} a_{n}^{2}=\sum_{K \geq 1} \sum_{n \in E_{K}} \frac{1}{K^{2}\left|E_{K}\right|}=\sum_{K \geq 1} \frac{1}{K^{2}}<\infty .
$$

ii) The series $\sum a_{n} g_{\lambda}(n x)$ is $L^{2}$-divergent. Indeed, using (5) and (8) :

$$
\begin{aligned}
\sum_{n \geq 1}\left(\sum_{k \geq 1} k^{-\lambda} a_{k n}\right)^{2} & \geq \sum_{K \geq 1} \sum_{n \in E_{K}^{\prime}}\left(\sum_{k \geq 1} k^{-\lambda} a_{k n}\right)^{2} \geq \sum_{K \geq 1} \sum_{n \in E_{K}^{\prime}}\left(\sum_{k \in \mathcal{P}_{K}} k^{-\lambda} a_{k n}\right)^{2} \\
& \geq \sum_{K \geq 1} \sum_{n \in E_{K}^{\prime}} \frac{1}{K^{2}\left|E_{K}\right|}\left(\sum_{k \in \mathcal{P}_{K}} k^{-\lambda}\right)^{2} \geq \sum_{K \geq 1}\left|E_{K}^{\prime}\right| \frac{K^{2}}{K^{2}\left|E_{K}\right|} \geq \sum_{K \geq 1} \frac{1}{2}=+\infty .
\end{aligned}
$$

Since the $a_{n}$ are positive, the conclusion comes from corollary 2.3 . 
iii) The series $\sum a_{n} g_{\lambda}(n x)$ is a.e-divergent. This requires longer computations. For a fixed $K \geq 1$, all $\left(X_{r, K}\right)_{1 \leq r \leq T_{K}}$ have the same law, due to the invariance of Lebesgue measure on $\mathbb{R} \backslash \mathbb{Z}$ under multiplication by an integer. They do not form a stationary process, but are nearly independent. Under our hypotheses, it is routine to check that the law of $\left(\sigma_{K}^{2} T_{K}\right)^{-1 / 2} \sum_{r=1}^{T_{K}} X_{r, K}$ is asymptotically normal.

In a first step, we compute the variance $\sigma_{K}^{2}$ and verify that it grows rapidly to infinity, as suggested by $i$ ). Via lemma 2.1 , we have :

$$
\begin{aligned}
\sigma_{K}^{2}=\operatorname{Var}\left(X_{1, K}\right) & =\frac{\zeta(2 \lambda)}{2} \frac{1}{K^{2}\left(m_{K}\right)^{l_{K}}} \sum_{n, m \in F_{1, K}}\left(\frac{n \wedge m}{n \vee m}\right)^{\lambda} \\
& =\frac{\zeta(2 \lambda)}{2} \frac{1}{K^{2}\left(m_{K}\right)^{l_{K}}} \sum_{1 \leq a_{j}, b_{j} \leq m_{K}, 1 \leq j \leq l_{K}} \prod_{j=1}^{l_{K}}\left(p_{j, K}\right)^{-\lambda\left|a_{j}-b_{j}\right|} \\
& =\frac{\zeta(2 \lambda)}{2} \frac{1}{K^{2}\left(m_{K}\right)^{l_{K}}} \prod_{j=1}^{l_{K}}\left(\sum_{a, b=1}^{m_{K}}\left(p_{j, K}\right)^{-\lambda|a-b|}\right) \\
& =\frac{\zeta(2 \lambda)}{2} \frac{1}{K^{2}\left(m_{K}\right)^{l_{K}}} \prod_{j=1}^{l_{K}}\left(m_{K}+2\left(p_{j, K}\right)^{-\lambda\left(m_{K}-1\right)} \sum_{k=1}^{m_{K}-1} k\left(p_{j, K}\right)^{\lambda(k-1)}\right) .
\end{aligned}
$$

For $x>1$, we have $\sum_{k=1}^{n-1} k x^{k-1}=\left((n-1) x^{n}-n x^{n-1}+1\right) /(x-1)^{2}=n x^{n-2}(1+o(1))$, when $x$ and $n$ are large. Inserting this in the previous calculations, we obtain, with a uniform $o(1)$ :

$$
\begin{aligned}
\sigma_{K}^{2} & =\frac{\zeta(2 \lambda)}{2} \frac{1}{K^{2}\left(m_{K}\right)^{l_{K}}} \prod_{j=1}^{l_{K}}\left(m_{K}+2 m_{K}\left(p_{j, K}\right)^{-\lambda}(1+o(1))\right) \\
& =\frac{\zeta(2 \lambda)}{2} \frac{1}{K^{2}} e^{\sum_{j=1}^{l_{K}} \log \left(1+2\left(p_{j, K}\right)^{-\lambda}(1+o(1))\right)} \\
& =\frac{\zeta(2 \lambda)}{2} \frac{1}{K^{2}} e^{2\left(\sum_{j=1}^{l_{K}}\left(p_{j, K}\right)^{-\lambda}\right)(1+o(1))} \geq e^{K},
\end{aligned}
$$

for large $K$, using (5).

We now establish the convergence :

$$
\left(\sigma_{K}^{2} T_{K}\right)^{-1 / 2} \sum_{r=1}^{T_{K}} X_{r, K} \rightarrow \mathcal{N}(0,1), \text { in law. }
$$

We write $E$ for the expectation under Lebesgue measure on $\mathbb{R} \backslash \mathbb{Z}$. Set $Y_{r, K}=\left(\sigma_{K}^{2} T_{K}\right)^{-1 / 2} X_{r, K}$ and $S_{N}=\sum_{r=1}^{N} Y_{r, K}$. For $1 \leq r \leq T_{K}$, introduce the finite partitions :

$$
\mathcal{F}_{r}=\left\{\left[k / q_{r, K},(k+1) / q_{r, K}\right), 0 \leq k<q_{r, K}\right\} .
$$

Each $Y_{r, K}$ being $\left(1 / q_{r, K}\right)$-periodic, for a bounded measurable $f$ :

$$
E\left(f\left(Y_{r, K}\right)\right)=E\left(f\left(Y_{r, K}\right) \mid \mathcal{F}_{r}\right)
$$

For $t \in \mathbb{R}$ and $2 \leq N \leq T_{K}$, we have :

$$
\begin{aligned}
& E\left(e^{i t S_{N}}\right)=E\left(e^{i t S_{N-1}} e^{i t Y_{N, K}}\right) \\
& =E\left(E\left(e^{i t S_{N-1}} \mid \mathcal{F}_{N}\right) e^{i t Y_{N, K}}\right)+E\left(\left(e^{i t S_{N-1}}-E\left(e^{i t S_{N-1}} \mid \mathcal{F}_{N}\right)\right) e^{i t Y_{N, K}}\right) \\
& \text { = } A+\text { B. }
\end{aligned}
$$


First of all, taking conditional expectation and using (12) :

$$
\begin{aligned}
A=E\left(E\left(e^{i t S_{N-1}} \mid \mathcal{F}_{N}\right) E\left(e^{i t Y_{N, K}} \mid \mathcal{F}_{N}\right)\right) & =E\left(E\left(e^{i t S_{N-1}} \mid \mathcal{F}_{N}\right) E\left(e^{i t Y_{N, K}}\right)\right) \\
& =E\left(e^{i t S_{N-1}}\right) E\left(e^{i t Y_{N, K}}\right) .
\end{aligned}
$$

Next :

$$
|B| \leq E\left(\left|e^{i t S_{N-1}}-E\left(e^{i t S_{N-1}} \mid \mathcal{F}_{N}\right)\right|\right) .
$$

The map $x \longmapsto e^{i t x}$ is $|t|$-Lipschitz. On each piece of $\mathcal{F}_{N}$ which contains no discontinuity of $S_{N-1}$, when counting the oscillation we have :

$$
\begin{aligned}
\left|e^{i t S_{N-1}}-E\left(e^{i t S_{N-1}} \mid \mathcal{F}_{N}\right)\right| & \leq \frac{|t|}{q_{N, K}} \frac{\left(\sigma_{K}^{2} T_{K}\right)^{-1 / 2}}{\left.\left(K\left(m_{K}\right)^{l_{K}}\right)\right)^{1 / 2}} \sum_{r=1}^{N-1}\left(m_{K}\right)^{l_{K}}\left(p_{1, K} \cdots p_{l_{K}, K}\right)^{m_{K}} q_{r, K} \\
& \leq|t|\left(\frac{\left(m_{K}\right)^{l_{K} / 2}}{K}\left(p_{1, K} \cdots p_{l_{K}, K}\right)^{m_{K}}\right) \frac{(N-1) q_{N-1, K}}{q_{N, K}}
\end{aligned}
$$

since $T_{K} \geq K$ and $\sigma_{K} \geq 1$ for large $K$, by (10). Next, $S_{N-1}$ is continuous on the interior of each segment of the partition whose step $^{-1}$ is $q_{1, K} \cdots q_{N-1, K}\left(p_{1, K} \cdots p_{l_{K}, K}\right)^{m_{K}}$. The total measure of the pieces of $\mathcal{F}_{N}$ which may contain a discontinuity of $S_{N-1}$ is bounded from above by:

$$
q_{1, K} \cdots q_{N-1, K}\left(p_{1, K} \cdots p_{l_{K}, K}\right)^{m_{K}} \frac{1}{q_{N, K}} \leq\left(p_{1, K} \cdots p_{l_{K}, K}\right)^{m_{K}} \frac{\left(q_{N-1, K}\right)^{N-1}}{q_{N, K}} .
$$

From (14), (15) and (16), we deduce that:

$$
|B| \leq 2(1+|t|)\left(p_{1, K} \cdots p_{l_{K}, K}\right)^{m_{K}}\left(1+\frac{\left(m_{K}\right)^{l_{K} / 2}}{K}\right) \frac{\left(q_{N-1, K}\right)^{N-1}}{q_{N, K}} .
$$

Using that for all $1 \leq N \leq T_{K}$, we have $\left|E\left(e^{i t Y_{N, K}}\right)\right| \leq 1$, when iterating the procedure with (13) and (14), we obtain via (6) :

$$
\begin{aligned}
\left|E\left(e^{i t S_{T_{K}}}\right)-\prod_{r=1}^{T_{K}} E\left(e^{i t Y_{r, K}}\right)\right| & \leq 2(1+|t|)\left(p_{1, K} \cdots p_{l_{K}, K}\right)^{m_{K}}\left(1+\frac{\left(m_{K}\right)^{l_{K} / 2}}{K}\right) \sum_{r=2}^{T_{K}} \frac{\left(q_{r-1, K}\right)^{r-1}}{q_{r, K}} \\
& \leq 2(1+|t|) \frac{1}{K} .
\end{aligned}
$$

As a consequence of (18), in order to show (11) we only need to focus on :

$$
\prod_{r=1}^{T_{K}} E\left(e^{i t Y_{r, K}}\right)=E\left(e^{i t Y_{1, K}}\right)^{T_{K}} .
$$

We now use the fact that for all $t \in \mathbb{R}$ :

$$
\left|e^{i t}-\left(1+i t-t^{2} / 2\right)\right| \leq \min \left\{|t|^{3} / 6,|t|^{2}\right\},
$$

which comes from $e^{i t}-\left(1+i t-t^{2} / 2\right)=i^{3} / 2 \int_{0}^{t}(t-s)^{2} e^{i s} d s=i^{2} \int_{0}^{t}(t-s)\left(e^{i s}-1\right) d s$. Via (20) and the property that $X_{1, K}$ has zero mean, we now deduce the following inequalities : 


$$
\begin{aligned}
\left|E\left(e^{i t Y_{1, K}}\right)-\left(1-\frac{t^{2}}{2 T_{K}}\right)\right| & \leq\left|E\left(e^{i t Y_{1, K}}-\left(1+i t Y_{1, K}-\frac{t^{2}}{2} Y_{1, K}^{2}\right)\right)\right| \\
& \leq E\left|\left(e^{i t Y_{1, K}}-\left(1+i t Y_{1, K}-\frac{t^{2}}{2} Y_{1, K}^{2}\right)\right)\right| \\
& \leq E\left(\min \left\{\left|t Y_{1, K}\right|^{3} / 6,\left|t Y_{1, K}\right|^{2}\right\}\right) .
\end{aligned}
$$

With $\varepsilon=\left(T_{K}\right)^{-5 / 12}$ and using (7), as well as $T_{K} \geq K$ and $\sigma_{K} \geq 1$ for large $K$ :

$$
\begin{aligned}
\left|E\left(e^{i t Y_{1, K}}\right)-\left(1-\frac{t^{2}}{2 T_{K}}\right)\right| & \leq \frac{|t|^{3}}{6} E\left(\left|Y_{1, K}\right|^{3} 1_{\left|Y_{1, K}\right| \leq \varepsilon}\right)+|t|^{2} E\left(\left|Y_{1, K}\right|^{2} 1_{\left|Y_{1, K}\right|>\varepsilon}\right) \\
& \leq \frac{|t|^{3}}{6\left(T_{K}\right)^{5 / 4}}+\frac{|t|^{2}}{\sigma_{K}^{2} T_{K}} E\left(\left|X_{1, K}\right|^{2} 1_{\left|X_{1, K}\right|>\left(T_{K}\right)^{1 / 12} \sigma_{K}}\right) \\
& \leq \frac{1}{T_{K}}\left(\frac{|t|^{3}}{6\left(T_{K}\right)^{1 / 4}}+\frac{|t|^{2}}{K \sigma_{K}^{2}}\right) \leq \frac{1}{T_{K}}\left(\frac{|t|^{3}}{6 K^{1 / 4}}+\frac{|t|^{2}}{K}\right) .
\end{aligned}
$$

Since $T_{K} \rightarrow+\infty$, as $K \rightarrow+\infty$, we deduce from (18), (19) and (21) that $E\left(e^{i t S_{T_{K}}}\right) \rightarrow e^{-t^{2} / 2}$, as $K \rightarrow+\infty$, for all $t \in \mathbb{R}$. This proves (11).

To conclude, for all $L \geq 1$ we choose $K_{L} \geq L$ so that :

$$
P\left(\left|S_{T_{K_{L}}}\right| \leq 1 / L^{2}\right) \leq 2 \int_{|t| \leq 1 / L^{2}} d \mathcal{N}(0,1)(t)=: \delta_{L} .
$$

Clearly $\sum_{L \geq 1} \delta_{L}<\infty$, so by Borel-Cantelli's lemma, for a.e $x$ when $L$ is large enough we have $\left|S_{T_{K_{L}}}\right| \geq 1 / L^{2}$. For such a $x$, using (10) and when $L$ is large enough :

$$
\left|Z_{K_{L}}\right|=\left|\frac{1}{\sqrt{T_{K_{L}}}} \sum_{r=1}^{T_{K_{L}}} X_{r, K_{L}}(x)\right|=\sigma_{K_{L}}\left|S_{T_{K_{L}}}\right| \geq \frac{e^{K_{L} / 2}}{L^{2}} \geq \frac{e^{L / 2}}{L^{2}} .
$$

Since partial sums $\sum_{K=1}^{N} Z_{K}(x)$ are partial sums of $\sum a_{n} g_{\lambda}(n x)$, this prevents $\sum a_{n} g_{\lambda}(n x)$ from converging at $x$. This completes the proof of item $i$ ) of theorem 1.1.

\section{Sufficient conditions for $L^{2}$ and a.e-convergence}

We take a finite sequence $\left(a_{n}\right)$ and write $\sum a_{n} g_{\lambda}(n x)=\sum b_{n} f_{n, \lambda}(x)$, where $\left(b_{n}\right)$ is also finite. By proposition 2.2 :

$$
\left\|\sum a_{n} g_{\lambda}(n x)\right\|^{2}=\left\|\sum b_{n} f_{n, \lambda}\right\|^{2} \leq \frac{\zeta(2 \lambda)}{2} \sum b_{n}^{2} \leq \frac{\zeta(2 \lambda)}{2} \sum_{n \geq 1}\left(\sum_{k \geq 1} k^{-\lambda}\left|a_{k n}\right|\right)^{2} .
$$

Set $\psi_{\lambda}(k)=k^{1-\lambda}(\log k)^{2}$ if $1 / 2<\lambda<1$ and $\psi_{1}(k)=\log k(\log \log k)^{1+\varepsilon}$, for some $\varepsilon>0$. For simplicity we write $\log (x)$ for $\max \{1, \log (x)\}$. Using Cauchy-Schwarz's inequality : 


$$
\begin{aligned}
\sum_{n \geq 1}\left(\sum_{k \geq 1} k^{-\lambda}\left|a_{k n}\right|\right)^{2} & =\sum_{k, k^{\prime} \geq 1}\left(k k^{\prime}\right)^{-\lambda} \sum_{n \geq 1}\left|a_{n k} a_{n k^{\prime}}\right| \leq \sum_{k, k^{\prime} \geq 1}\left(k k^{\prime}\right)^{-\lambda}\left(\sum_{n \geq 1} a_{n k}^{2}\right)^{1 / 2}\left(\sum_{n \geq 1} a_{n k^{\prime}}^{2}\right)^{1 / 2} \\
& \leq\left[\sum_{k \geq 1} k^{-\lambda}\left(\sum_{n \geq 1} a_{n k}^{2}\right)^{1 / 2}\right]^{2} \leq\left(\sum_{k \geq 1} k^{-\lambda} \frac{1}{\psi_{\lambda}(k)}\right)\left(\sum_{k \geq 1} k^{-\lambda} \psi_{\lambda}(k) \sum_{n \geq 1} a_{n k}^{2}\right) \\
& \leq C_{\varepsilon} \sum_{n \geq 1} a_{n}^{2} \sum_{k \mid n} k^{-\lambda} \psi_{\lambda}(k) .
\end{aligned}
$$

We first consider the case $1 / 2<\lambda<1$. Remark that $0<2 \lambda-1<1$. Using a classical upper-bound for $\sum_{k \mid n} k^{2 \lambda-1}$, cf Krätzel [13], we have for any $\delta>0$ :

$$
\begin{aligned}
\sum_{k \mid n} k^{-\lambda} \psi_{\lambda}(k) & =\sum_{k \mid n} k^{1-2 \lambda}(\log k)^{2} \leq(\log n)^{2} \sum_{k \mid n} k^{1-2 \lambda} \leq(\log n)^{2} n^{1-2 \lambda} \sum_{k \mid n} k^{2 \lambda-1} \\
& \leq(\log n)^{2} n^{1-2 \lambda} C_{\delta} n^{2 \lambda-1} e^{\frac{(1+\delta)(\log n)^{1-(2 \lambda-1)}}{(1-(2 \lambda-1)) \log \log n}} \leq C_{\delta}^{\prime} n^{\frac{(1+2 \delta)(\log n)^{1-2 \lambda}}{2(1-\lambda) \log \log n}}
\end{aligned}
$$

In the situation when $\lambda=1$, we have :

$$
\sum_{k \mid n} k^{-1} \psi_{1}(k) \leq \psi_{1}(n) \sum_{k \mid n} k^{-1}=\psi_{1}(n) n^{-1} \sum_{k \mid n} k .
$$

We use this time the inequality $\sum_{k \mid n} k \leq C n \log \log n$, see again [13]. As a result, for any $\varepsilon>0$ there is a constant $C_{\varepsilon}>0$ such that for any sequence $\left(a_{n}\right)$ :

$$
\left\{\begin{array}{c}
\left\|\sum a_{n} g_{\lambda}(n x)\right\|^{2} \leq C_{\varepsilon} \sum a_{n}^{2} n^{\frac{(1+\varepsilon)(\log n)-(2 \lambda-1)}{2(1-\lambda) \log \log n}}, \text { when } 1 / 2<\lambda<1, \\
\left\|\sum a_{n}\{n x\}\right\|^{2} \leq C_{\varepsilon} \sum a_{n}^{2} \log n(\log \log n)^{2+\varepsilon}, \text { when } \lambda=1 .
\end{array}\right.
$$

These properties imply the $L^{2}$-convergence of $D_{\lambda}$-series under the assumptions of theorem 1.1.

We turn to the question of the a.e-convergence of $D_{\lambda}$-series. The second item of theorem 1.1 is a consequence of inequalities (23) and of the following proposition. The latter is an adaptation of a method due to Rademacher [16] for the study of series based on a general orthonormal family.

\section{Proposition 4.1}

Let $\left(a_{n}\right)_{n \geq 1}$ and $(\varphi(n))_{n \geq 1}$ be such that $\sum a_{n}^{2} \varphi(n)(\log n)^{2}<\infty$ and for any $M \leq N$ :

$$
\left\|\sum_{n=M}^{N} a_{n} g_{\lambda}(n x)\right\|^{2} \leq \sum_{n=M}^{N} a_{n}^{2} \varphi(n) .
$$

Then $\sum a_{n} g_{\lambda}(n x)$ converges a.e.

Proof of the proposition :

We can suppose that log is the logarithm in base 2. Let $S(n)(x)=\sum_{1 \leq k \leq n} a_{k} g_{\lambda}(k x)$. For $m<n$, introduce the notations :

$$
S(m, n)(x)=\sum_{m \leq k<n} a_{k} g_{\lambda}(n x) \text { and } \sigma_{l}(m, n)=\sum_{m \leq k<n} a_{k}^{2} \varphi(k)(\log k)^{l}, \text { for } l \in\{0,1,2\}
$$


Step 1. We show that $\left(S\left(2^{n}\right)(x)\right)$ converges for a.e $x$. Let $0<N<n$. We have :

$$
\begin{aligned}
\int_{0}^{1} \sum_{N \leq r<n} S\left(2^{r}, 2^{n}\right)^{2}(x) d x & \leq \sum_{N \leq r<n} \sigma_{0}\left(2^{r}, 2^{n}\right)=\sum_{N \leq r<n} \sum_{s=r}^{n-1} \sigma_{0}\left(2^{s}, 2^{s+1}\right) \\
& \leq \sum_{s=N}^{n-1}(s-N+1) \sigma_{0}\left(2^{s}, 2^{s+1}\right) \\
& \leq \sum_{s=N}^{n-1} s \sigma_{0}\left(2^{s}, 2^{s+1}\right) \leq \sigma_{1}\left(2^{N}, 2^{n}\right) .
\end{aligned}
$$

By Markov's inequality, $\sum_{N \leq r<n} S\left(2^{r}, 2^{n}\right)^{2}(x) \leq \sigma_{1}\left(2^{N}, 2^{n}\right)^{2 / 3}$ for all $x$ in a Borel set $E_{N, n}$ with :

$$
\lambda\left(E_{N, n}\right) \geq 1-\sigma_{1}\left(2^{N}, 2^{n}\right)^{1 / 3} \geq 1-\sigma_{1}\left(2^{N}, \infty\right)^{1 / 3} .
$$

In particular, for $x \in E_{N, n}$ and all $N \leq r<n$, we have $S\left(2^{r}, 2^{n}\right)(x) \leq \sigma_{1}\left(2^{N}, 2^{n}\right)^{1 / 3}$. Define a set $E_{N, n}^{\prime}$ by the condition that for all $N \leq r \leq r^{\prime}<n$ :

$$
S\left(2^{r}, 2^{r^{\prime}}\right)(x) \leq 2 \sigma_{1}\left(2^{N}, \infty\right)^{1 / 3} .
$$

Since $E_{N, n} \subset E_{N, n}^{\prime}$, we have $\lambda\left(E_{N, n}^{\prime}\right) \geq 1-\sigma_{1}\left(2^{N}, \infty\right)^{1 / 3}$. Fixing $N$, the $E_{N, n}^{\prime}$ are monotonic in $n$.

The set $D_{N}$ defined by the condition that for all $N \leq r \leq r^{\prime}, S\left(2^{r}, 2^{r^{\prime}}\right)(x) \leq 2 \sigma_{1}\left(2^{N}, \infty\right)^{1 / 3}$ has therefore a measure $\lambda\left(D_{N}\right) \geq 1-\sigma_{1}\left(2^{N}, \infty\right)^{1 / 3}$. Since $\lambda\left(D_{N}\right) \rightarrow 1$, as $N \rightarrow+\infty$, we deduce that $\lambda\left(\lim \sup D_{N}\right)=1$. If $x \in \limsup D_{N}$, the sequence $\left(S\left(2^{n}\right)(x)\right)$ clearly satisfies the Cauchy criterion, so converges. This concludes step 1.

Step 2. To complete the proof, we show that a.e $\sup _{2^{r}<n<2^{r+1}}\left|S\left(2^{r}, n\right)(x)\right| \rightarrow 0$, as $r \rightarrow+\infty$. Let $2^{r}<n<2^{r+1}$ and decompose $n$ in base 2 :

$$
n=2^{r}+\sum_{l=1}^{r} \theta_{l} 2^{r-l}, \text { with } \theta_{l} \in\{0,1\} .
$$

Then :

$$
S\left(2^{r}, n\right)=\sum_{l=1}^{r} S\left(2^{r}+\sum_{m=1}^{l-1} \theta_{m} 2^{r-m}, 2^{r}+\sum_{m=1}^{l} \theta_{m} 2^{r-m}\right) .
$$

By convexity :

$$
\begin{aligned}
S\left(2^{r}, n\right)^{2} & \leq r \sum_{l=1}^{r} S\left(2^{r}+\sum_{m=1}^{l-1} \theta_{m} 2^{r-m}, 2^{r}+\sum_{m=1}^{l} \theta_{m} 2^{r-m}\right)^{2} \\
& \leq r \sum_{l=1}^{r} \sum_{h=0}^{2^{r-l}-1} S\left(2^{r}+h 2^{l}, 2^{r}+h 2^{l}+2^{l-1}\right)^{2}=: T(r) .
\end{aligned}
$$

The quantity $T(r)$ is independent on $2^{r}<n<2^{r+1}$. Next : 


$$
\begin{aligned}
\int_{0}^{1} T(r)(x) d x & =r \sum_{l=1}^{r} \sum_{h=0}^{2^{r-l}-1} \int_{0}^{1} S\left(2^{r}+h 2^{l}, 2^{r}+h 2^{l}+2^{l-1}\right)^{2}(x) d x \\
& \leq r \sum_{l=1}^{r} \sum_{h=0}^{2^{r-l}-1} \sigma_{0}\left(2^{r}+h 2^{l}, 2^{r}+h 2^{l}+2^{l-1}\right) \\
& \leq r \sum_{l=1}^{r} \sigma_{0}\left(2^{r}, 2^{r+1}\right)=r^{2} \sigma_{0}\left(2^{r}, 2^{r+1}\right) \leq \sigma_{2}\left(2^{r}, 2^{r+1}\right) .
\end{aligned}
$$

Fix $N$ and let $r \geq N$. By Markov's inequality, for $x$ in a Borel set $F_{r}(N)$ of Lebesgue measure $\lambda\left(F_{r}(N)\right) \geq 1-\sigma_{2}\left(2^{r}, 2^{r+1}\right) / \sigma_{2}\left(2^{N}, \infty\right)^{2 / 3}$, we have :

$$
\sup _{2^{r}<n<2^{r+1}} S\left(2^{r}, n\right)^{2}(x) \leq T(r) \leq \sigma_{2}\left(2^{N}, \infty\right)^{2 / 3} .
$$

Let $G_{N}=\cap_{r \geq N} F_{r}(N)$. Then $\lambda\left(G_{N}\right) \geq 1-\sum_{r \geq N} \sigma_{2}\left(2^{r}, 2^{r+1}\right) / \sigma_{2}\left(2^{N}, \infty\right)^{2 / 3}=1-\sigma_{2}\left(2^{N}, \infty\right)^{1 / 3}$. For $x \in G_{N}$ :

$$
\forall r \geq N, \sup _{2^{r}<n<2^{r+1}}\left|S\left(2^{r}, n\right)(x)\right| \leq \sigma_{2}\left(2^{N}, \infty\right)^{1 / 3} .
$$

As $\lambda\left(G_{N}\right) \rightarrow 1$, we get $\lambda\left(\lim \sup G_{N}\right)=1$. If $x \in \lim \sup G_{N}$, then $\sup _{2^{r}<n<2^{r+1}}\left|S\left(2^{r}, n\right)(x)\right|$ tends to 0 , as $r \rightarrow \infty$. This concludes step 2 and the proof of the proposition.

\section{Particular classes where $L^{2}$-convergence is true}

We consider the proof of theorem 1.2.

\subsection{Proof of $i)$}

Let $\left(n_{k}\right)$ be lacunary in the sense that $n_{k+1} / n_{k} \geq c>1$ and $\left(a_{k}\right) \in l^{2}$. We first consider the upper bound. We can assume the sequence $\left(a_{k}\right)$ finite. By lemma 2.1, the $L^{2}$-norm of $(2 / \zeta(2 \lambda))^{1 / 2} \sum a_{k} g_{\lambda}\left(n_{k} x\right)$ is given by :

$$
\sum_{k, l \geq 1} a_{k} a_{l}\left(\frac{n_{k} \wedge n_{l}}{n_{k} \vee n_{l}}\right)^{\lambda}=\sum a_{k}^{2}+2 \sum_{k<l} a_{k} a_{l} \frac{\left(n_{k} \wedge n_{l}\right)^{2 \lambda}}{\left(n_{k} n_{l}\right)^{\lambda}} .
$$

Using Cauchy-Schwarz's inequality, the second term is bounded by :

$$
\begin{aligned}
\sum_{k<l}\left|a_{k} a_{l}\right| \frac{\left(n_{k} \wedge n_{l}\right)^{2 \lambda}}{\left(n_{k} n_{l}\right)^{\lambda}} \leq \sum_{k<l}\left|a_{k} a_{l}\right| \frac{n_{k}^{2 \lambda}}{\left(n_{k} n_{l}\right)^{\lambda}} & \leq \sum_{k<l}\left|a_{k} a_{l}\right| c^{-\lambda(l-k)} \leq \sum_{k \geq 1}\left|a_{k}\right| \sum_{l \geq 1} c^{-\lambda l}\left|a_{k+l}\right| \\
& \leq\left(\sum_{n \geq 1} a_{k}^{2}\right)^{1 / 2}\left(\sum_{k \geq 1}\left(\sum_{l \geq 1} c^{-\lambda l}\left|a_{k+l}\right|\right)^{2}\right)^{1 / 2}
\end{aligned}
$$

Next, still via Cauchy-Schwarz's inequality : 


$$
\begin{aligned}
\sum_{k \geq 1}\left(\sum_{l \geq 1} c^{-\lambda l}\left|a_{k+l}\right|\right)^{2} & =\sum_{l, l^{\prime} \geq 1} c^{-\lambda\left(l+l^{\prime}\right)} \sum_{k \geq 1}\left|a_{k+l} a_{k+l^{\prime}}\right| \\
& \leq \sum_{l, l^{\prime} \geq 1} c^{-\lambda\left(l+l^{\prime}\right)}\left(\sum_{k \geq 1} a_{k+l}^{2}\right)^{1 / 2}\left(\sum_{k \geq 1} a_{k+l^{\prime}}^{2}\right)^{1 / 2} \\
& \leq\left(\sum_{l \geq 1} c^{-\lambda l}\left(\sum_{k \geq 1} a_{k+l}^{2}\right)^{1 / 2}\right)^{2} \leq \frac{1}{\left(c^{\lambda}-1\right)^{2}} \sum_{k \geq 1} a_{k}^{2} .
\end{aligned}
$$

As a consequence :

$$
\sum_{k<l}\left|a_{k} a_{l}\right| \frac{\left(n_{k} \wedge n_{l}\right)^{2 \lambda}}{\left(n_{k} n_{l}\right)^{\lambda}} \leq \frac{1}{\left(c^{\lambda}-1\right)} \sum_{k \geq 1} a_{k}^{2}
$$

Since $1+2 /\left(c^{\lambda}-1\right)=\left(c^{\lambda}+1\right) /\left(c^{\lambda}-1\right)$, this completes the proof of the upper-bound.

For the lower bound, one can also suppose that $\left(a_{k}\right)$ is finite. We have $\sum a_{k} g_{\lambda}\left(n_{k} x\right)=\sum b_{k} f_{k, \lambda}$, where $\left(b_{k}\right)$ is also finite. By proposition 2.2 :

$$
\left\|\sum a_{k} g_{\lambda}\left(n_{k} x\right)\right\|^{2}=\left\|\sum b_{k} f_{k, \lambda}\right\|^{2} \geq \frac{1}{2} \sum b_{k}^{2} .
$$

Fixing $0<\varepsilon<1-(2 \lambda)^{-1}$, giving $2 \lambda(1-\varepsilon)>1$ :

$$
\begin{aligned}
\sum a_{k}^{2}=\sum_{k \geq 1}\left(\sum_{l \geq 1} l^{-\lambda} \mu(l) b_{l n_{k}}\right)^{2} & \leq \sum_{k \geq 1}\left(\sum_{l \geq 1} l^{-2 \lambda(1-\varepsilon)} \mu(l)^{2}\right)\left(\sum_{l \geq 1} l^{-2 \lambda \varepsilon} b_{l n_{k}}^{2}\right) \\
& \leq \prod_{i \geq 1}\left(1+p_{i}^{-2 \lambda(1-\varepsilon)}\right)\left(\sum_{l \geq 1} b_{l}^{2} \sum_{k, n_{k} \mid l}\left(\frac{n_{k}}{l}\right)^{2 \lambda \varepsilon}\right) \\
& \leq \prod_{i \geq 1}\left(\frac{1-p_{i}^{-4 \lambda(1-\varepsilon)}}{1-p_{i}^{-2 \lambda(1-\varepsilon)}}\right)\left(\sum_{m \geq 0} c^{-2 \lambda \varepsilon m}\right) \sum_{l \geq 1} b_{l}^{2} \\
& \leq \frac{\zeta(2 \lambda(1-\varepsilon))}{\zeta(4 \lambda(1-\varepsilon))}\left(1-c^{-2 \lambda \varepsilon}\right)^{-1} \sum_{l \geq 1} b_{l}^{2} .
\end{aligned}
$$

To complete the proof, we first use that $\zeta(4 \lambda(1-\varepsilon)) \geq \zeta(4 \lambda)$ and $\zeta(2 \lambda(1-\varepsilon)) \leq 1+1 /(2 \lambda(1-\varepsilon)-1)$. Set $\varepsilon=\rho(1-1 /(2 \lambda))$, with $0<\rho<1$. We have :

$$
\left(1+\frac{1}{2 \lambda(1-\varepsilon)-1}\right) \frac{1}{1-c^{-2 \lambda \varepsilon}} \leq \frac{2 \lambda}{2 \lambda-1} \frac{1}{(1-\rho)\left(1-c^{-(2 \lambda-1) \rho}\right)} .
$$

Minimizing in $\rho$, we take $\rho=1 /\left(1+\ln c^{2 \lambda-1}\right)$. We finally use the inequality $1-e^{-x} \geq(1-1 / e) x$, for $0 \leq x \leq 1$, giving $\left(1-c^{-(2 \lambda-1) \rho}\right) \geq(1-1 / e)(1-\rho)$.

Concerning a.e-convergence, we can now apply proposition 4.1 with $\varphi=1$. 


\subsection{Proof of $i i)$}

We start from (22). For $n$ with $|\operatorname{supp}(n)| \leq N$ and any $0<\delta<2 \lambda-1$, we have :

$$
\sum_{k \mid n} k^{-\lambda} \psi_{\lambda}(k) \leq C_{\delta} \sum_{k \mid n} k^{1-2 \lambda+\delta} \leq C_{\delta} \prod_{p \mid n, p \in \mathcal{P}}\left(1-p^{1-2 \lambda+\delta}\right)^{-1} \leq C_{\delta} \prod_{i=1}^{N}\left(1-p_{i}^{1-2 \lambda+\delta}\right)^{-1} .
$$

For the lower bound, we use $\left\|\sum b_{n} f_{n, \lambda}\right\|^{2} \geq(1 / 2) \sum b_{n}^{2}$, by proposition 2.2. Next :

$$
\sum a_{k}^{2}=\sum_{k \geq 1}\left(\sum_{l \geq 1} l^{-\lambda} \mu(l) b_{l k}\right)^{2} \leq \sum_{k \geq 1}\left(\sum_{l \geq 1} l^{-\lambda}\left|b_{l k}\right|\right)^{2} \leq C_{\varepsilon} \sum_{n \geq 1} b_{n}^{2} \sum_{k \mid n} k^{-\lambda} \psi_{\lambda}(k),
$$

when proceeding in the same way as for (22). We then conclude as above, using the fact that $b_{n}=0$ when $|\operatorname{supp}(n)|>N$. For a.e-convergence, we apply proposition 4.1 with $\varphi=1$.

\subsection{Proof of $i i i)$}

Using the remark after lemma 2.1 we only need to focus on $\left(b_{n}\right)$. Set $b_{i, n}=b_{p_{i}^{n}}$. Multiplicativity implies that the $\left(b_{i, n}\right)_{i, n \geq 1}$ entirely determine the sequence $\left(b_{n}\right)$. Via corollary (2.3), we show that:

$$
\sum_{n \geq 1}\left(\sum_{k \geq 1} k^{-\lambda} b_{k n}\right)^{2}<+\infty
$$

Each term in this series is finite, by Cauchy-Schwarz's inequality. We first claim the equivalence $\left(b_{n}\right) \in l^{2} \Leftrightarrow \sum_{i \geq 1} \sum_{n \geq 1} b_{i, n}^{2}<+\infty$. Indeed, using that $b_{1}=1$, we have :

$$
\sum_{n \geq 1} b_{n}^{2}=1+\sum_{k \geq 1} \sum_{1 \leq i_{1}<\cdots<i_{k}} \sum_{u_{1} \geq 1, \cdots, u_{k} \geq 1} b_{i_{1}, u_{1}}^{2} \cdots b_{i_{k}, u_{k}}^{2}=\prod_{i \geq 1}\left(1+\sum_{n \geq 1} b_{i, n}^{2}\right) .
$$

This proves the claim.

For technical reasons, up to considering $\tilde{b}_{i, n}=b_{i, n}+1 /(i n),(i, n) \geq 1$, and the corresponding multiplicative sequence $\left(\tilde{b}_{n}\right)$, which satisfies $\left(\tilde{b}_{n}\right) \in l^{2} \Leftrightarrow\left(b_{n}\right) \in l^{2}$, we assume that $b_{i, n}>0$ for all indices $(i, n) \geq 1$. Decomposing in prime factors $n=p_{i_{1}}^{u_{1}} \cdots p_{i_{k}}^{u_{k}}$, with $k=0$ if $n=1$, and using multiplicativity :

$$
\begin{aligned}
\sum_{n \geq 1}\left(\sum_{l \geq 1} l^{-\lambda} b_{l n}\right)^{2}= & \sum_{k \geq 0} \sum_{1 \leq i_{1}<\cdots<i_{k}} \sum_{u_{1} \geq 1, \cdots, u_{k} \geq 1} \\
& \prod_{j \notin\left\{i_{1}, \cdots, i_{k}\right\}}\left(1+\sum_{m \geq 1} p_{j}^{-\lambda m} b_{j, m}\right) \times \prod_{l=1, \cdots, k}\left(\sum_{v_{l} \geq 0} p_{i_{l}}^{-\lambda v_{l}} b_{i_{l}, u_{l}+v_{l}}\right) .
\end{aligned}
$$

The first product term is uniformly bounded from above since for a constant $C$ :

$$
\sum_{j, m \geq 1} p_{j}^{-\lambda m} b_{j, m} \leq\left(\sum_{j, m \geq 1} p_{j}^{-2 \lambda m}\right)^{1 / 2}\left(\sum_{j, m \geq 1} b_{j, m}^{2}\right)^{1 / 2} \leq C\left(\sum_{j \geq 1} p_{j}^{-2 \lambda}\right)^{1 / 2}\left(\sum_{j, m \geq 1} b_{j, m}^{2}\right)^{1 / 2}<+\infty .
$$

To prove (24), it remains to check the finiteness of : 


$$
\sum_{k \geq 0} \sum_{1 \leq i_{1}<\cdots<i_{k}} \prod_{l=1}^{k}\left[\sum_{u_{l} \geq 1}\left(\sum_{v_{l} \geq 0} p_{i_{l}}^{-\lambda v_{l}} b_{i_{l}, u_{l}+v_{l}}\right)^{2}\right]=\prod_{i \geq 1}\left[1+\sum_{u \geq 1}\left(\sum_{v \geq 0} p_{i}^{-\lambda v} b_{i, u+v}\right)^{2}\right] .
$$

It is equivalent to showing :

$$
\sum_{i \geq 1, u \geq 1}\left(\sum_{v \geq 0} p_{i}^{-\lambda v} b_{i, u+v}\right)^{2}<+\infty
$$

Set $c_{i, n}=p_{i}^{\lambda n} \sum_{v \geq n} p_{i}^{-\lambda v} b_{i, v}$, which is finite by Cauchy-Schwarz's inequality. We thus verify that $\sum_{i \geq 1, n \geq 1} c_{i, n}^{2}<+\infty$. Fixing $0<\varepsilon<1-2^{-\lambda}$, we prove below that for all $i \geq 1$ :

$$
\begin{aligned}
\sum_{n \geq 1} c_{i, n}^{2} & \leq \frac{1}{\varepsilon^{2}\left(1-(1-\varepsilon)^{-2} p_{i}^{-2 \lambda}\right)} \sum_{n \geq 1} c_{i, n}^{2}\left(1-\frac{c_{i, n+1}}{p_{i}^{\lambda} c_{i, n}}\right)^{2} \\
& \leq \frac{1}{\varepsilon^{2}\left(1-(1-\varepsilon)^{-2} 2^{-2 \lambda}\right)} \sum_{n \geq 1} b_{i, n}^{2}
\end{aligned}
$$

Since $\sum_{i, n \geq 1} b_{i, n}^{2}<\infty$, this brings the conclusion.

Fix $i \geq 1$ and introduce $\mathcal{C}=\left\{n \geq 1,\left|1-c_{i, n+1} /\left(p_{i}^{\lambda} c_{i, n}\right)\right|<\varepsilon\right\}$. We claim that if $\mathcal{C}$ is infinite, then it does not contain all large integers. Indeed, if $n \in \mathcal{C}$, then $c_{i, n+1} \geq(1-\varepsilon) p_{i}^{\lambda} c_{i, n}$, since $c_{i, n+1}<p_{i}^{\lambda} c_{i, n}$. If $\mathcal{C}$ contains some interval $\left[n_{0},+\infty\right)$, then for $n \geq n_{0}:$

$$
\sum_{v \geq 0} b_{i, v+n} p_{i}^{-\lambda v}=p_{i}^{\lambda n} \sum_{v \geq n} b_{i, v} p_{i}^{-\lambda v} \geq p_{i}^{\lambda n}(1-\varepsilon)^{n-n_{0}} \sum_{v \geq n_{0}} b_{i, v} p_{i}^{-\lambda v} .
$$

However $\sum_{v \geq n_{0}} b_{i, v} p_{i}^{-\lambda v}$ is fixed and $>0$, since $b_{i, v}>0$. As $p_{i}^{\lambda}(1-\varepsilon)>1$, a contradiction is given by Cauchy-Schwarz's inequality, because the left-hand side is bounded from above by :

$$
\left(\sum_{v \geq 0} p_{i}^{-2 \lambda v}\right)^{1 / 2}\left(\sum_{v \geq 0} b_{i, n+v}^{2}\right)^{1 / 2} \leq\left(\sum_{v \geq 0} p_{i}^{-2 \lambda v}\right)^{1 / 2}\left(\sum_{v \geq 0} b_{i, v}^{2}\right)^{1 / 2}<+\infty .
$$

Decompose now into disjoint intervals $\mathcal{C}=\cup_{k \geq 1}\left[a_{k}, b_{k}\right]$ and write in a disjoint union :

$$
\{n \geq 1\}=\left[a_{1}, b_{1}\right] \cup\left[a_{1}^{\prime}, b_{1}^{\prime}\right] \cup \cdots \cup\left[a_{k}, b_{k}\right] \cup\left[a_{k}^{\prime}, b_{k}^{\prime}\right] \cup \cdots
$$

Notice that the first interval $\left[a_{1}, b_{1}\right]$ may be empty, whereas the other ones are not, and that the collection of $\left(\left[a_{k}, b_{k}\right],\left[a_{k}^{\prime}, b_{k}^{\prime}\right]\right)_{k}$ may be finite. We have :

$$
\sum_{n \notin \mathcal{C}, n \notin\left\{a_{k}^{\prime}, k \geq 1\right\}} c_{i, n}^{2}\left(1-\frac{c_{i, n+1}}{p_{i}^{\lambda} c_{i, n}}\right)^{2} \geq \varepsilon^{2} \sum_{n \notin \mathcal{C}, n \notin\left\{a_{k}^{\prime}, k \geq 1\right\}} c_{i, n}^{2} .
$$

Also :

$$
\sum_{k \geq 1} \sum_{l=a_{k}}^{a_{k}^{\prime}} c_{i, l}^{2}\left(1-\frac{c_{i, l+1}}{p_{i}^{\lambda} c_{i, l}}\right)^{2} \geq \varepsilon^{2} \sum_{k \geq 1} c_{i, a_{k}^{\prime}}^{2}
$$

Observe finally that : 


$$
\sum_{l=a_{k}}^{a_{k}^{\prime}} c_{i, l}^{2} \leq c_{i, a_{k}^{\prime}}^{2} \sum_{m \geq 0}(1-\varepsilon)^{-2 m} p_{i}^{-2 \lambda m} \leq\left(1-(1-\varepsilon)^{-2} p_{i}^{-2 \lambda}\right)^{-1} c_{i, a_{k}^{\prime}}^{2} .
$$

Combining (27), (28) and (29), we get (26). This completes the proof of this item.

Acknowledgements. We thank Zoltan Buczolich about the first point of theorem 1.1.

\section{References}

[1] Balazard, M. and Saias, E., Notes on the Riemann zeta-function. IV, Adv. Math. 188 (2004), no. 1, 69-86.

[2] Bourgin, D. And Mendel, C., Orthonormal sets of periodic functions of the type $\{f(n x)\}$, Trans. Amer. Math. Soc. 57, 1945, 332-363.

[3] De la Bretèche, R. And Tenenbaum, G., Séries trigonométriques à coefficients arithmétiques, J. Anal. Math. 92 (2004), 1-79.

[4] Carleson, L., On convergence and growth of partial sums of Fourier series, Acta Math. 116 (1966), 135-157.

[5] Davenport, H., On some infinite series involving arithmetical functions, Quart. J. Math., vol 8, pp. 8-13 (1937).

[6] Davenport, H., On some infinite series involving arithmetical functions II, Quart. J. Math., vol 8, pp. 313-320 (1937).

[7] Fefferman, C., Pointwise convergence of Fourier series, Ann. of Math. 98 (1973), 551-571.

[8] Hardy, G. And Littlewood, J., Some problems of Diophantine approximation : the analytic properties of certain Dirichlet's series associated with the distribution of numbers of modulus unity, Trans. Cambridge Philos. Soc., vol. 22 (1923), 519-533.

[9] Hartman, P., On a class of arithmetical Fourier series, Amer. J. Math. vol. 60 (1938), 66-74.

[10] Haukkanen, P., Wang, J. And Sillanp, J., On Smith's determinant, Linear Algebra Appl. 258 (1997), 251-269.

[11] Hedenmalm, H., Lindqvist, P. And Seip., K., A Hilbert space of Dirichlet series and systems of dilated functions in $L^{2}(0,1)$, Duke Math. J., vol. 86, pp. 1-37 (1997).

[12] Jaffard, S., On Davenport expansions, Fractal geometry and applications : a jubilee of Benoît Mandelbrot. Part 1, 273-303, Proc. Sympos. Pure Math., 72, Part 1, Amer. Math. Soc., Providence, RI, 2004.

[13] Krätzel, E., Zahlentheorie, Berlin (1981).

[14] Lang, S., Introduction to Diophantine approximation, Springer-Verlag (1995).

[15] Lindqvist, P. And Seip., K., Note on some greatest common divisor matrices, Acta Arith. 84 (1998), no. 2, 149-154.

[16] Rademacher, H., Einige Sätze über Reihen von allgemeinen Orthogonalfunktionen, Math. Ann. 87 (1922), 112-138.

[17] Wintner, A., Diophantine approximation and Hilbert's space, Amer. J. Math., vol. 66 (1944), 564-578.

Laboratoire d'Analyse et de Mathématiques Appliquées, Université Paris Xit, Faculté des Sciences et Technologies, 61 avenue du Général de Gaulle, 94010 Créteil Cedex, France E-mail address : bremont@univ-paris12.fr 